\title{
Mapping Texts to Multidimensional Emotional Space: Challenges for Dataset Acquisition in Sentiment Analysis
}

\author{
Alexander Kalinin 1[0000-0002-0012-1692], Anastasia Kolmogorova ${ }^{10000-0002-6425-2050]}$, Galina \\ Nikolaeva ${ }^{1[0000-0002-3740-2922]}$, Alina Malikova ${ }^{1[0000-0002-3438-1839]}$ \\ ${ }^{1}$ Siberian Federal University, 79 Svobodny pr.,660041 Krasnoyarsk, Russian Federation \\ \{aakalinin, avkolmogorova, gnikolaeva, avmalikova\}@sfu-kras.ru
}

\begin{abstract}
The cornerstone for any sentiment analysis research is labeled data and its acquisition. Canonical corpuses for this task contain different reviews (movies, restaurants) where sentiment can be derived from reviewer's explicit rating of a reviewed item. Ratings go with supplied comments, which are used as text samples and ratings are converted into labels. Usually emotion labels come in binary form like "negativelpositive".

This simplistic approach works well when we are dealing with binary emotional model, but it turns to fail when we are dealing with more complex emotional models like "Pleasure-Arousal-Dominance (PAD)" or Lövheim's Cube, when we collect data from various sources and of different types (fiction books, social networks conversations, blog posts etc.) or when we delegate labeling to external assessors.

In the article, we describe which methodological problems we faced while collecting dataset for sentiment analysis backed by Lövheim's Cube - emotional model that represents an emotion as a point in three-dimensional space of balance of three monoamines (Dopamine, Serotonin and Noradrenaline).

These problems include the choice of necessary metadata to be collected along with text and labels, choice of tools used for labeling and survey design.
\end{abstract}

Keywords: Sentiment analysis, Emotion label, Lövheim's Cube.

\section{Lövheim's Cube Emotional Model}

The goal of the research is to investigate linguistic representations of emotions in context of Lövheim's Cube of Emotions in Russian language and their practical aspects for example in use of sentiment analysis related tasks.

We have chosen this emotional model because it is neurologically funded. Major psychological theories of emotions provide phenomenological views of emotions - usually emotions are defined subjectively depending of what a person feels. Such approaches are vague from formalist point of view and can be hardly used computerrelated fields. Otherwise, Lövheim's approach provides formal representation of emo- 
tions as functions of neurotransmitters, which are responsible for neuro-somatic response towards some stimulus. This function takes objective parameters to be the arguments [2] and these parameters are mixtures or proportions of three monoamine neurotransmitters: serotonin, dopamine and noradrenaline. The balance of these monoamines forms a three-dimensional space represented by the following visual mode (Fig. $1)$ :

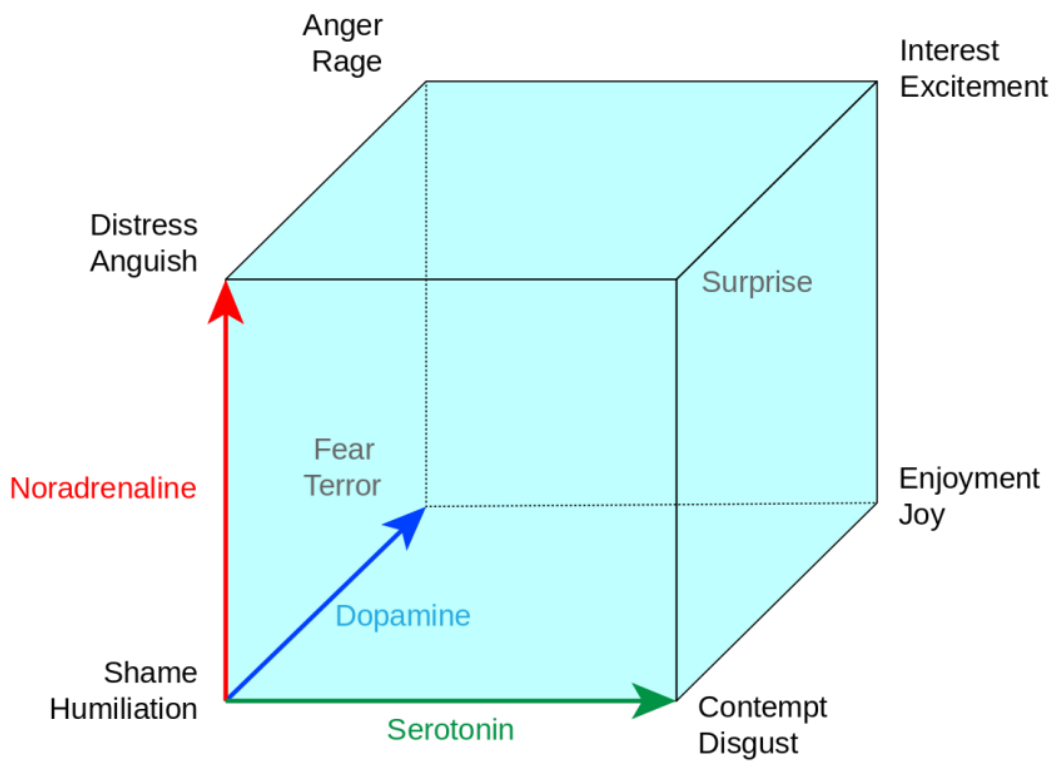

Fig. 1. Lövheim's cube of emotions

Such approach for modelling emotions numerically is also used outside neurology. It is used for creating intellectual emotional agents in robotics or computer games.

\section{Data Acquisition for Sentiment Analysis}

For this particular research or other research that deals with sentiment analysis, we need labeled or annotated data. A big amount of randomized samples from different sources makes it feasible for conduction of deep and reliable research in sphere of relations between a text and emotional state that stand behind it on big dataset.

Canonical corpora which are available for English and Russian mostly contain datasets with binary labels - "positive" or "negative". Such corpora can be used for building models for text classification, like reviews, forums and social networks comments, but can't be used for deep research in emotion-text interconnection as they are bound to binary scale, and emotions are certainly can't be reduced to positivelnegative ones. 
Unfortunately, there are few large corpora for Russian language with labels beyond binary scale, and there are no corpora labeled according to Lövheim's model neither for Russian nor for English. So in our research we faced the need of collecting such a corpus. Certainly such data should be collected from different sources with help of assessor with different social and demographic background. For this task the solution appeared to be crowdsourcing platforms, which can facilitate speed of label with help of large community.

If problem with assessors can be considered to be solved, the problem upon how to label data remains open - two main questions arise:

- How to map texts with Lövheim's Cube emotional model inventory

- Which tools to provide for such a mapping.

The straightforward way is to use labels - there are 8 vertices of the cube that can be used as corresponding labels. When can add a "neutral" label to this set and suggest an assessor to choose the label that he thinks is the most suitable for the text sample.

But in such approach we potentially lose information for cases with intermediate emotions. The advantage of the Lövheim's Cube emotional model is that it provides non-discrete continuous estimation of emotion states, as emotions themselves are not discrete - particular emotion can be a mix of anger and disgust, or excitement and surprise on so on, and each component in emotion can be expressed higher or lower. In discrete approach, we have to invent names for such "intermediate" emotions, but Lövheim's model allows us to use numeric coordinates. For example: what could be the name for an emotion between surprise and disgust? No need to name this complex emotion, in terms of Lövheim's Cube model it can be referenced as $(0.5,0,1)$ where first coordinate is for noradrenaline level, second is for dopamine and the third is for serotonin. That problem is which tools we should provide to map text to this coordinates.

\section{$3 \quad$ Mapping Emotions to Point in Space}

Major feature of Lövheim's cube are coordinate axes, which correspond to degree of presence of three monoamines - noradrenaline, serotonin and dopamine. Minimal degree of all monoamines corresponds to ShamelHumiliation; the maximum degree corresponds to Interest ไExcitement. It's quite straightforward to ask an assessor to set coordinates of emotion in three coordinates, for example with help of scroll bars (Fig. 2): 
Please, adjust the scrollbar of monoamines

to posistion you think mostly suites the text

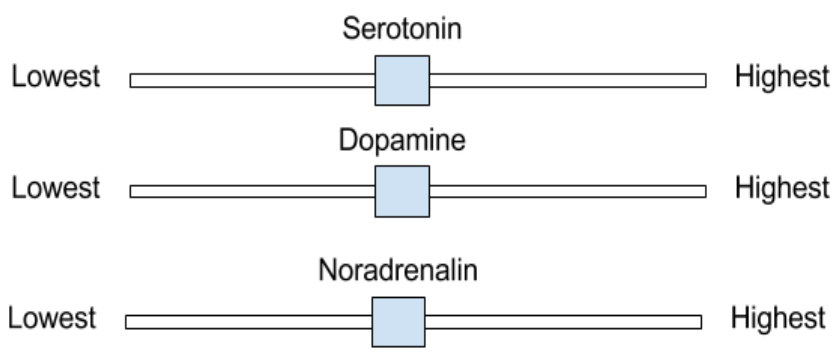

Fig. 2. Scroll-bars for assessing text with monoamines expressions

With help of scrollbars we can map their positions to the point to explicitly setting its coordinates. But here we face with problem that an ordinary assessor is not aware for such unknown thing as monoamines expression. Moreover neurological details and necessary pre-teaching of those concepts for assessor can be a serious distraction while data acquisition and so may lead to low quality of assessments.

The workaround here maybe to interpret monoamines degree of expression in terms familiar to an assessor. For example, noradrenalin is responsible for arousal, dopamine is for reinforcement, and serotonin is for self-confidence [7]. But nevertheless such terms are too abstract and quite neurology-domain specific. An ordinary assessor can hardly evaluate "reinforcement" or "arousal" of a sentiment in text. We should step out from verbalizing monoamines functions in this task and search for other variants that should definitely engage terms familiar to an assessor to operate.

\subsection{Mapping Emotions Using Supporting Cube Diagonals}

For representing emotional space in terms familiar for an assessor we can use oppositions between emotions situated on cube's vertices. For such oppositions we can use cube diagonals shown on Fig. 3: 


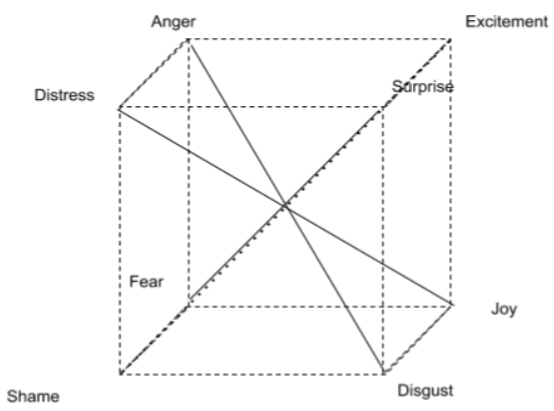

Fig. 3. Supporting diagonals of Lövheim's Cube

In such case we have four oppositions

- Distress - Enjoyment

- Rage - Disgust

- Shame - Excitement

- Fear - Surprise

Here we can see that these emotion pairs forms four dichotomies and at their extreme points monoamines' balance is inverted - fear, for example, is a negative form of surprise, rage is aggressive antonym of disgust etc. These terms are familiar for assessors, and they can reliably estimate their subjective impact of a text sample. Moreover with such approach respondents may scale the emotional expression to differentiate for example "very aggressive" text from "moderately aggressive" one.

With these oppositions we may ask assessors to adjust each slide bar to positions they think mostly corresponds to. If one of oppositions is not expressed in text the scrollbar should remain in central (neutral) position. Possible interface for this kind of task is presented on Fig. 4:

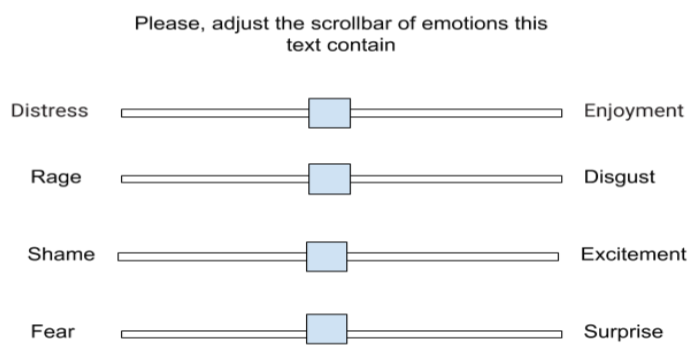

Fig. 4. Scrollbars with diagonal oppositions 
Such approach also provides opportunity to point "secondary emotions", in case when text sample is mostly about fear sentiment, but an assessor want to mention slight impact of rage. In case of discrete labels when only one answer is allowed it's impossible to do so.

To process such responds with supporting diagonals we should treat each scrollbar position as vector. For this task we need to shift the center of axes to the center of cube that can be view a neutral state. The distance from cube's center to its vertices is one. This will allow us to treat each opposition as $(-1,1)$ period and to map a slide-bar position to a vector which angle coincides with corresponding diagonal and the magnate that is equal to value chosen for a given emotional oppositions. Scrollbar that remains in neutral position are considered to be null vectors with null magnitude.

To get the final estimation of the text we sum up the vectors. For example scrollbars position on Fig. 5 tells us that an assessor considered estimated text sample to express much surprise with a bit of disgust. His estimates from scrollbar can be converted to vectors on Fig. 6 the resulting vector points on Surprise domain.

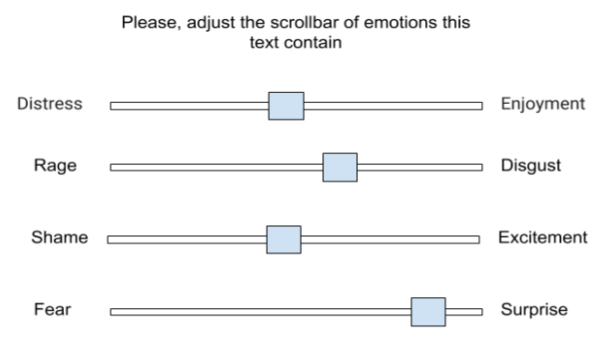

Fig. 5. Possible configuration of scrollbar

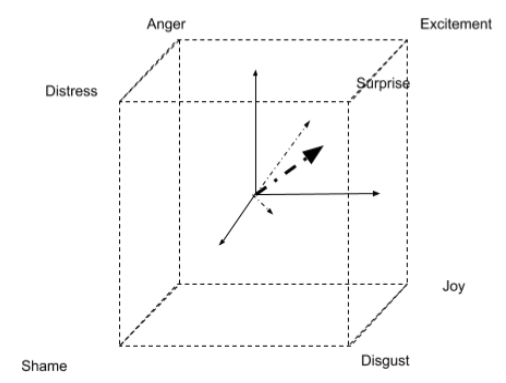

Fig. 6. Vector representation of response in Fig. 5 


\section{Conclusion and Discussion}

At the time of writing, this approach towards data acquisition in such a continuous wat is under development. This concept is believed to be very promising as it suggests stepping out from viewing text sentiment as discrete objects and moving towards more complex continuous way of accessing and representing text sentiments. Of course there several problems:

The first one is validation of Lövheim's theory itself - because of novelty and relation with neurology it has to be validated by neurological tools like tomogram or medical tests. By now, the status of his theory does not enable wide campaigns for biological studies. His Cube remains only a possible model.

The second problem for our approach is handling corner cases when an assessor decides to toggle scrollbars to random extreme position. To prevent this we might add some vector metrics including angular deviations in assessor's response.

The third problem is "user-friendliness" of our approach. In spite of provide complex answers, toggling four different sidebar is still more difficult than just choosing one label, and here we have to engage UXIUI competences. In addition, a question of correct instructions remains unresolved.

\section{References}

1. Bermingham, A., Smeaton A.: Classifying sentiment in microblogs: is brevity an advantage? Proceedings of the 19th ACM international conference on Information and knowledge management. ACM (2010). doi: 10.1145/1871437.1871741

2. Galitsky, B., Chen, H., Du, S.: Inversion of Forum Content Based on Authors' Sentiments on Product Usability. AAAI Spring Symposium: Social Semantic Web: Where Web 2.0 Meets Web 3.0 (2009).

3. Garrett, J.: The Elements of User Experience: User-Centered Design for the Web and Beyond. 2nd edn. New Riders, USA (2011). doi: 10.1524/icom.2.1.44.19040

4. Hu, M., Bing L.: Mining opinion features in customer reviews. AAAI 4(4), 755-760 (2004).

5. Jitviriya, W., Koike, M., Hayashi, E.: Emotional model for robotic system using a self-organizing map combined with Markovian model. Journal of Robotics and Mechatronics. 27(5), 563-570 (2015). doi: 10.20965/jrm.2015.p0563

6. Larue, O.: The emergence of (artificial) emotions from cognitive and neurological processes. Biologically Inspired Cognitive Architectures vol. 4, 55-68 (2013). doi: 10.1016/j.bica.2013.01.001

7. Lövheim, H.: A new three-dimensional model for emotions and monoamine neurotransmitters. Med Hypotheses 78, 341-348 (2012). doi: 10.1016/j.mehy.2011.11.016

8. Marcus, A.: Design, User Experience, and Usability: Design Discourse. In: Marcus, A. (ed.) CONFERENCE 2015, LNCS, vol. 9186, 672 P. Springer, Heidelberg (2015). doi: $10.1007 / 978-3-319-20886-2$ 